\title{
Metabolic Health, Obesity, and Renal Function: 2013-2018 National Health and Nutrition Examination Surveys
}

\author{
Kathleen E. Adair ${ }^{1, *(\mathbb{C}}$, Rodney G. Bowden ${ }^{2}$, LesLee K. Funderburk ${ }^{1}{ }^{\oplus}$, Jeffrey S. Forsse ${ }^{1}\left(\mathbb{D}\right.$ and Kelly R. Ylitalo ${ }^{2, *}$ \\ 1 Department of Health, Human Performance, and Recreation, Robbins College of Health and Human Sciences, \\ Baylor University, One Bear Place \#97313, Waco, TX 76798, USA; leslee_funderburk@baylor.edu (L.K.F.); \\ jeff_forsse@baylor.edu (J.S.F.) \\ 2 Department of Public Health, Robbins College of Health and Human Sciences, Baylor University, One Bear \\ Place \#97343, Waco, TX 76798, USA; rodney_bowden@baylor.edu \\ * Correspondence: katie_adair1@baylor.edu (K.E.A.); kelly_ylitalo@baylor.edu (K.R.Y.); \\ Tel.: +1-254-710-4271 (K.R.Y.)
}

Citation: Adair, K.E.; Bowden, R.G.; Funderburk, L.K.; Forsse, J.S.; Ylitalo, K.R. Metabolic Health, Obesity, and Renal Function: 2013-2018 National Health and Nutrition Examination Surveys. Life 2021, 11, 888. https:// doi.org/10.3390/life11090888

Academic Editors: Emilio Nardi and Giuseppe Mule

Received: 9 July 2021

Accepted: 13 August 2021

Published: 28 August 2021

Publisher's Note: MDPI stays neutral with regard to jurisdictional claims in published maps and institutional affiliations.

Copyright: (c) 2021 by the authors. Licensee MDPI, Basel, Switzerland. This article is an open access article distributed under the terms and conditions of the Creative Commons Attribution (CC BY) license (https:// creativecommons.org/licenses/by/ $4.0 /)$.
Abstract: Rising rates of metabolic syndrome, obesity, and mortality from chronic kidney disease (CKD) have prompted further investigation into the association between metabolic phenotypes and CKD. Purpose: To report the frequency of strictly defined metabolic phenotypes, renal function within each phenotype, and individual risk factors associated with reduced renal function. We utilized the 2013-2018 National Health and Nutrition Examination Surveys (NHANES) and complex survey sample weighting techniques to represent 220 million non-institutionalized U.S. civilians. Metabolic health was defined as having zero of the risk factors defined by the National Cholesterol Education Program with the exception of obesity, which was defined as BMI $\geq 30 \mathrm{~kg} / \mathrm{m}^{2}$ in non-Asians and $\mathrm{BMI} \geq 25 \mathrm{~kg} / \mathrm{m}^{2}$ in Asians. The metabolically healthy normal (MUN) phenotype comprised the highest proportion of the population $(38.40 \%)$, whereas the metabolically healthy obese (MHO) was the smallest $(5.59 \%)$. Compared to the MHN reference group, renal function was lowest in the strictly defined MUN $(B=-9.60, p<0.001)$ and highest in the MHO $(B=2.50, p>0.05)$, and this persisted when an increased number of risk factors were used to define metabolic syndrome. Systolic blood pressure had the strongest correlation with overall eGFR $(r=-0.25, p<0.001)$, and individuals with low HDL had higher renal function compared to the overall sample. The MUN phenotype had the greatest association with poor renal function. While the $\mathrm{MHO}$ had higher renal function, this may be due to a transient state caused by renal hyperfiltration. Further research should be done to investigate the association between dyslipidemia and CKD.

Keywords: chronic kidney disease; CKD; metabolic phenotypes; obesity; metabolic risk factors

\section{Introduction}

In the past three decades the incidence of end-stage renal disease (ESRD) has increased by approximately $93 \%$ [1], and chronic kidney disease (CKD) is the third fastest growing cause of premature mortality [2]. CKD is a costly [3] and burdensome health issue that more often results in premature mortality than in ESRD [4]. Steady increases in rates of metabolic syndrome and obesity are occurring in the U.S., with both conditions recently exceeding previous levels at 34.2\% [5] and 42.6\% [6] of the U.S. population, respectively. Approximately 15\% of U.S. adults are estimated to have CKD [7], and it is likely that the prevalence will increase given the associations of CKD with metabolic risk factors, such as type 2 diabetes mellitus (T2D), hypertension (HTN) [7], and obesity [7-9].

Metabolic phenotypes, which take into account metabolic risk factors and obesity, have been utilized to assess the risk of various outcomes, such as cardiovascular disease (CVD), mortality, and CKD. A recent meta-analysis by Alizadeh et al. [10] analyzed nine prospective cohort studies that compared CKD risk among metabolic phenotypes and 
found that the metabolically healthy obese (MHO) and the metabolically unhealthy normal weight (MUN), termed the "intriguing" phenotypes, shared a similarly elevated risk of developing CKD, with pooled relative risks (RR) of 1.55 and 1.58, respectively. This meta-analysis included studies with primarily Asian populations, limiting generalizability, and the definitions of the metabolic phenotypes varied, hindering the comparability between studies.

Prior research in the area of metabolic phenotyping has reported equivocal findings regarding the number of risk factors used to define the "metabolically unhealthy" status, with the most strict definitions determining that one or more [11-14] risk factors should be considered unhealthy and the more liberal ranging from two or more $[15,16]$ to three or more [17-20] risk factors. A recent publication by Lavie et al. [21] proposed a harmonized definition that classifies the metabolically healthy phenotype as having zero of the four metabolic risk factors. This rationale is based on the notion that individuals with hyperglycemia, dyslipidemia, and/or hypertension cannot be considered "healthy" and therefore should not be classified as such [21]. Several large studies [13,14,22] have previously used this definition, and several more $[11,12,23]$ have adopted it since it was first proposed by Lavie et al.

In this study, our primary purpose was to report the prevalence of the strict metabolic phenotypes in the U.S. population utilizing NHANES data and complex survey sample weighting. Additionally, we reported the association between renal function and the metabolic phenotypes, utilizing the three most common definitions of metabolic health Lastly, we identified and reported the individual risk factors associated with reduced renal function.

\section{Materials and Methods}

The institutional review board at Baylor University determined the present study exempt from review [IRB ID\# 1505514-1]. The project was classified as non-human subjects research because the data are deidentified and widely available for use via the CDC. Survey sample weighting, which includes a complex, four-stage, probability cluster, was utilized for the present analyses. Sample weighting procedures are outlined by the National Center for Health Statistics Estimating and Weighting Procedures documents [24,25].

\subsection{Study Sample}

The National Health and Nutrition Examination Surveys (NHANES) are studies conducted in 2-year cycles by the Centers for Disease Control and Prevention (CDC). The NHANES design utilizes complex survey sample weighting procedures to produce nationally representative health statistics for the U.S. The population sampled by NHANES was limited to civilian, non-institutionalized individuals who lived within the U.S. at the time of sampling. In order to increase the reliability and precision of weighted estimates for underrepresented populations, oversampling of individuals 60 and over, African Americans, Asians, and Hispanics was routinely conducted. Sample weights were assigned to each individual in a sample in order to extrapolate the results to a represent all non-institutionalized U.S. civilians.

The inclusion criteria for the study required subjects to have completed one of the three most recently published cycles of the NHANES survey, which included the 20132014, 2015-2016, and 2017-2018 cycles. Inclusion criteria further required that subjects be between the ages of 18 and 79 and have complete study information to classify metabolic and renal health. The upper age limit was chosen because individuals 80 years and older in the NHANES dataset are top coded at 80 for subject deidentification; therefore, age cannot be controlled for over 79 years. The biological markers used to identify metabolic and renal health included fasting glucose, fasting triglycerides, high-density lipoprotein (HDL), blood pressure, body mass index (BMI), age, sex, race, and serum creatinine (SCr) value. Subjects were excluded from the study if they reported pregnancy and/or tested 
positive for a pregnancy test. Additionally, individuals who reported being on dialysis in the 12 months prior to the study were excluded from analysis.

The initial study sample included 29,400 subjects. A total of 12,594 did not meet the inclusion criteria for age, 190 were pregnant at the time of the study, 9988 did not have biological markers sufficient to classify metabolic and/or renal health, and 18 subjects reported use of dialysis. The final sample that was analyzed included 6610 U.S. citizens, which was representative of a population size of 220,388,819 individuals after the NHANES survey sample weights were applied.

\subsection{Definition of Metabolic Phenotypes}

Metabolic risk factors were defined using criteria from the National Cholesterol Education Program's (NCEP) Adult Treatment Panel III (ATP III) [26], with the exception of obesity, which was defined as a BMI $>30 \mathrm{~kg} / \mathrm{m}^{2}$ for all non-Asian individuals and a BMI $>25 \mathrm{~kg} / \mathrm{m}^{2}$ for all individuals identified as Asian $[16,18,20]$. Metabolically healthy or unhealthy status was determined by the four remaining metabolic risk factors: hyperglycemia, which was defined as a fasting glucose $\geq 100 \mathrm{mg} / \mathrm{dL}$ or prescription medication for hyperglycemia; the two dyslipidemia criteria, which were defined as a fasting triglyceride $\geq 150 \mathrm{mg} / \mathrm{dL}$, a high-density lipoprotein level $<40 \mathrm{mg} / \mathrm{dL}$ for males, $<50 \mathrm{mg} / \mathrm{dL}$ for females, or a prescription medication for dyslipidemia; and hypertension was defined as a resting systolic blood pressure $>130 \mathrm{mmHg}$, a resting diastolic blood pressure $>85 \mathrm{mmHg}$, or prescription medication for hypertension (Table 1). In the primary analyses, metabolic health was defined as the absence of all metabolic risk factors in Table 1, excluding the measure of obesity. Therefore, the metabolically healthy normal weight (MHN) phenotype was defined as the absence of all metabolic risk factors and absence of obesity; metabolically healthy obese (MHO) required the absence of all metabolic risk factors and presence of obesity; metabolically unhealthy normal weight (MUN) required the presence of one or more metabolic risk factors and absence of obesity; and the metabolically unhealthy obese (MUO) required the presence of one or more metabolic risk factors and presence of obesity.

Table 1. Criteria for metabolic risk factors and metabolic phenotypes.

\begin{tabular}{ccl}
\hline Category & Classification & \multicolumn{1}{c}{ Values } \\
\hline & Obesity & Non-Asian $\mathrm{BMI} \geq 30 \mathrm{~kg} / \mathrm{m}^{2}$, Asian BMI $\geq 25 \mathrm{~kg} / \mathrm{m}^{2}$ \\
Hetabolic Risk Factor & Fasting glucose $\geq 100 \mathrm{mg} / \mathrm{dL}$ or Rx \\
& Dyslipidemia & $\mathrm{TG} \geq 150 \mathrm{mg} / \mathrm{dL}$ or $\mathrm{Rx}$ \\
& (2nd criteria) & $>130 \mathrm{mmHg}$ systolic or $>85 \mathrm{mmHg}$ diastolic or Rx \\
\hline & Hypertension & Non-obese and $<1$ metabolic risk factor \\
Metabolic & MHN & Obese and $<1$ metabolic risk factor \\
Phenotype & MHO & Non-obese and $>1$ metabolic risk factor \\
& MUN & Obese and $>1$ metabolic risk factor
\end{tabular}

Metabolic syndrome is defined by the NCEP ATP III (2005 Revision) guidelines [26]. BMI is calculated as weight (kg) divided by height $\left(\mathrm{m}^{2}\right)$. Rx, prescription medication for given risk factor; TG, triglycerides; HDL, high density lipoprotein; M, males; F, females; MHN, metabolically healthy normal weight; MHO, metabolically healthy obese; MUN, metabolically unhealthy normal weight; MUO, metabolically unhealthy obese.

\subsection{Renal Outcome Measures}

Renal function was calculated using the Chronic Kidney Disease Epidemiology Collaboration (CKD-EPI) equation [27]:

$$
\left.\mathrm{eGFR}=141 \times \min \left(\frac{S C r}{\kappa}, 1\right)^{\alpha} \times \max \left(\frac{S C r}{\kappa}, 1\right)^{-1.209} \times 0.993^{\text {Age }} \times 1.018 \text { (if female }\right) \times 1.159 \text { (if Black) }
$$

where eGFR is the estimated glomerular filtration rate, $\mathrm{SCr}$ is serum creatinine collected as part of the standard biochemistry profile using the DxC 800 chemistry analyzer, $\kappa$ is 0.7 if female or 0.9 if male, $\alpha$ is -0.329 if female or -0.411 if male, min is the minimum of 
$\frac{S C r}{\kappa}$ or 1 , and max is the maximum of $\frac{S C r}{\kappa}$ or 1 . The CKD-EPI equation has been reported to be more accurate than the MDRD equation in individuals with higher GFRs [27]. CKD was defined as an eGFR $<60 \mathrm{~mL} / \mathrm{min} / 1.73 \mathrm{~m}^{2}$ (categories G3 to G5) and/or an albumin to creatinine ratio $\geq 30 \mathrm{mg} / \mathrm{g}$ [28]. All individuals who reported use of dialysis in the 12 months prior to the study were excluded from the analyses.

\subsection{Questionnaires, Examinations, and Laboratory Data}

The NHANES interview-style questionnaires include demographic, socioeconomic, dietary, health history, and lifestyle information. Age, binary sex, and race/ethnicity were determined by questionnaires that were asked in the home by trained interviewers using the Computer-Assisted Personal Interview (CAPI) system. Total caloric intake was determined using two 24-h dietary interviews, and a composite variable was created to average dietary intake for two-day samples. Dietary intakes were assessed on all days of the week, with the 2 measurements typically separated by 3 days. Eighteen percent of the dietary intake information was missing in the present sample. Subsample weights (WTDR2D sample weight variable) were utilized to marginally adjust for race and Hispanic origin, age group, sex, weekday-weekend categories, and day-two non-responders. SES was determined by dividing family (or individual) income by the poverty guidelines defined by the U.S. federal government. Subjects who fell at or below $100 \%$ of the poverty level for the given year, which is a common criterion for determining eligibility in federal assistance programs [29], were considered low SES. Physical activity (PA), reported in minutes per day and number of days per week, was classified using the guidelines from the PA Guidelines Advisory Committee Report [30]. Individuals were considered physically active if they took part in $\geq 150$ min of moderate-intensity recreational PA per week, $\geq 75$ min of vigorous-intensity recreational PA per week, or an equivalent combination of the two [30,31]. Implausible PA values were reported in this sample; therefore, values $\geq 4 \mathrm{~h}$ per day of recreational PA were top-coded at $4 \mathrm{~h}$. There was $49 \%$ missingness in the PA variable. Subjects were considered smokers if they have smoked at least 100 cigarettes in their lifetime or if they reported having smoked in the past 5 days. All others were considered non-smokers. International Classification of Diseases, Tenth Revision (ICD-10) codes were used to determine prescription medication $(\mathrm{Rx})$ information for hyperglycemia (R73, E11, E11.2, E11.2P, E11.4, and E11.P), hypercholesterolemia (E78.0, E78.0P, and E78.1), and hypertension (I10 and I10.P).

The NHANES examination includes anthropometric measures, blood pressure, blood panels, and urinalysis. BMI was calculated using height, which is measured in meters $(\mathrm{m})$ on a calibrated stadiometer; and weight, which is measured on a calibrated digital weight scale or a portable scale. The waist circumference (WC) was taken at the level of the uppermost lateral border of the iliac crest and reported in centimeters $(\mathrm{cm})$ for each subject. Three consecutive measures of blood pressure (BP) are taken after a 5-min seated rest period. In cases where the BP measurement was interrupted or incomplete, a fourth measure was taken and reported. The present analysis reported the mean blood pressure for each subject by averaging the three available systolic and diastolic blood pressures. Fasting blood samples were taken and reported for blood lipids and blood glucose. The lipid sample was analyzed using the Roche/Hitachi Cobas 6000 analyzer (Roche Diagnostics, Indianapolis, IN, USA), and the serum low-density lipoprotein (LDL), expressed in milligrams per deciliter $(\mathrm{mg} / \mathrm{dL})$ was calculated utilizing the Friedewald calculation [32]. Fasting plasma glucose was analyzed using the Roche Cobas C311 system. Serum high-sensitivity C-reactive protein (hs-CRP) levels were measured beginning in the 2015-2016 cycle of NHANES; therefore, 36.7\% of the sample has missing values for this variable since it was not collected in the 2013-2014 cycle. The Beckman UniCel ${ }^{\circledR}$ DxC 600 and 600i Synchron chemistry analyzers (Beckman Coulter, Brea, CA, USA) were used to measure hs-CRP in the 2015-2016 cycle, and the Roche Cobas 6000 was used in the 2017-2018 cycles. The homeostatic model assessment of insulin resistance (HOMAIR), which is a method utilized to quantify insulin resistance and beta-cell function, was 
calculated using the following equation [33]: fasting glucose $(\mathrm{mmol} / \mathrm{L}) \times$ fasting insulin (microU $/ \mathrm{mL}) / 22.5$. The albumin to creatinine ratio was reported in $\mathrm{mg} / \mathrm{g}$ utilizing the fluorescein immunoassay by Sequoia-Turner Digital Fluorometer, Model 450 (SequoiaTurner Corporation, Mountain View, CA, USA) to determine urinary albumin, and the Roche Cobas 6000 Analyzer was used to measure urinary creatinine.

The percentage of glycated hemoglobin $(\mathrm{HbA} 1 \mathrm{c})$ was not reported in the present study because its value was determined by questionnaire rather than a blood panel. Alcohol intake was not analyzed because the reporting method changed during the 2017-2018 cycle and could not be compared to prior surveys.

\subsection{Statistical Analysis}

Statistical analyses were conducted using SAS version 9.4 (SAS Institute Inc., Cary, NC, USA). A DOMAIN statement was used to analyze the subpopulation meeting study inclusion criteria. Masked variance pseudo-primary sampling unit (PSU), masked variance pseudo-stratum, and fasting subsample 2-year mobile examination center (MEC) weights from NHANES were used for sample weighting. Unweighted demographic information was represented using means and standard deviations (SD) for continuous variables or frequencies and percentages $(n, \%)$ for categorical variables. Weighted demographic data were reported for the total sample and metabolic phenotypes using a weighted mean and standard error of the mean (SE) for continuous variables or a percentage (\%) and the standard error of percent (SE) for categorical variables. Simple regression analyses of weighted data were used to identify statistical differences between continuous demographic variables. Chi-square $\left(\chi^{2}\right)$ tests were used to identify statistical differences between categorical demographic variables. Pearson's product moment correlation coefficients $(r)$ were used to identify correlations between two continuous variables. Linear regression models with complex survey sample weighting were used to determine the influence of metabolic phenotype on renal function. In model 1 , we considered one metabolic risk factor to be unhealthy; in Model 2, we considered 2 risk factors to be unhealthy, and in Model 3, we considered 3 risk factors to be unhealthy. For all analyses, the level of significance was set a priori at $\alpha=0.05$.

\section{Results}

The weighted sample population of 6610 subjects who met the study inclusion criteria represented 220,388,819 non-institutionalized U.S. civilians. The weighted and unweighted demographic data are represented in Table 2. The prevalence of obesity was $42.49 \%$, with an average BMI of 29.4 ( $\mathrm{SE}=0.18$ ). The prevalence of individuals with at least one metabolic risk factor (excluding obesity) was $75.30 \%$, and only $19.11 \%$ of the sample was metabolically healthy and non-obese. The most frequent metabolic phenotype was the MUN phenotype (38.40\%) followed by the MUO (36.90\%), and the phenotype that represented the smallest proportion of the sample was the $\mathrm{MHO}(5.59 \%)$. The metabolically unhealthy phenotypes were more likely to be male, older age, current or former smokers, have metabolic risk factors, and have poor renal function, whereas the metabolically healthy individuals tended to have higher HDL-cholesterol and reported that they engaged in greater amounts of recreational physical activity. The obese phenotypes were more likely to be female, non-Hispanic (NH) Black Americans, and have higher levels of hs-CRP, whereas the normal-weight individuals were more likely to be NH White or NH Asian despite more conservative obesity cutoff values for NH Asians. There was no statistically significant difference between phenotypes for daily caloric intake or frequency of individuals with low SES. 
Table 2. Demographic information for subsample from the 2013-2018 National Health and Nutrition Examination Survey.

\begin{tabular}{|c|c|c|c|c|c|c|c|}
\hline & $\begin{array}{l}\text { Unweighted Total } \\
\quad(n=6610)\end{array}$ & $\begin{array}{l}\text { Weighted Total } \\
(n=220,388,819)\end{array}$ & MHN (19.11\%) & MHO (5.59\%) & $\begin{array}{c}\text { MUN } \\
(38.40 \%)\end{array}$ & $\begin{array}{c}\text { MUO } \\
(36.90 \%)\end{array}$ & \multirow{2}{*}{$p$-Value } \\
\hline & Mean (SD) & Mean (SE) & Mean (SE) & Mean (SE) & Mean (SE) & Mean (SE) & \\
\hline Age (years) & $47.03(17.04)$ & $45.61(0.37)$ & $35.72(0.58)$ & $36.22(0.86)$ & $49.31(0.57)$ & $48.31(0.52)$ & $<0.001$ \\
\hline BMI $\left(\mathrm{kg} / \mathrm{m}^{2}\right)$ & $29.4(7.33)$ & $29.40(0.18)$ & $23.42(0.13)$ & $33.48(0.28)$ & $25.33(0.09)$ & $36.10(0.24)$ & $<0.001$ \\
\hline Waist Circumference $(\mathrm{cm})$ & 99.35 (17.15) & $99.83(0.43)$ & $83.75(0.40)$ & $105.93(0.84)$ & $92.25(0.30)$ & $115.19(0.50)$ & $<0.001$ \\
\hline Caloric Intake (Kcal/day) & $2048(853)$ & $2087(17)$ & $2083(43)$ & $2032(56)$ & $2123(25)$ & $2058(27)$ & 0.207 \\
\hline Fasting Glucose (mg/dL) & $110.71(37.50)$ & $107.74(0.49)$ & $91.54(0.26)$ & $92.41(0.38)$ & $108.35(0.65)$ & $117.83(0.83)$ & $<0.001$ \\
\hline Triglycerides (mg/dL) & $115.59(112.38)$ & $114.16(1.70)$ & $66.21(1.21)$ & $75.35(1.81)$ & $117.73(2.03)$ & $141.14(3.20)$ & $<0.001$ \\
\hline HDL (mg/dL) & $53.75(16.11)$ & $54.29(0.36)$ & $64.82(0.65)$ & $59.06(1.07)$ & $54.49(0.53)$ & $47.90(0.37)$ & $<0.001$ \\
\hline Systolic BP (mmHg) & $123.31(18.00)$ & $121.41(0.29)$ & $110.05(0.40)$ & $113.55(0.49)$ & $122.64(0.44)$ & $127.19(0.37)$ & $<0.001$ \\
\hline Diastolic BP (mmHg) & $70.13(12.28)$ & $70.30(0.29)$ & $65.32(0.32)$ & $68.11(0.62)$ & $70.49(0.42)$ & $73.01(0.33)$ & $<0.001$ \\
\hline $\mathrm{eGFR}\left(\mathrm{mL} / \mathrm{min} / 1.73 \mathrm{~m}^{2}\right)$ & $97.7(22.17)$ & $97.16(0.50)$ & $103.93(0.91)$ & $106.44(1.25)$ & $94.34(0.64)$ & $95.19(0.61)$ & $<0.001$ \\
\hline hs-CRP (mg/L) & $4.15(8.25)$ & $3.80(0.18)$ & $1.39(0.07)$ & $4.49(0.52)$ & $2.92(0.26)$ & $5.68(0.28)$ & $<0.001$ \\
\hline $\mathrm{ACR}(\mathrm{mg} / \mathrm{g})$ & $41.66(291.46)$ & $29.14(2.78)$ & $16.70(2.33)$ & $10.49(3.07)$ & $23.34(2.93)$ & $44.45(6.20)$ & $<0.001$ \\
\hline HOMA-IR & $4.22(8.52)$ & $3.77(0.10)$ & $1.35(0.03)$ & $2.51(0.10)$ & $2.63(0.07)$ & $6.43(0.22)$ & $<0.001$ \\
\hline $\mathrm{SCr}(\mathrm{mg} / \mathrm{dL})$ & $0.86(0.28)$ & $0.86(0.00)$ & $0.83(0.00)$ & $0.84(0.01)$ & $0.86(0.00)$ & $0.87(0.01)$ & $<0.001$ \\
\hline \multirow[t]{2}{*}{ BUN (mg/dL) } & $13.74(5.24)$ & $13.84(0.12)$ & $13.03(0.16)$ & $12.61(0.31)$ & $14.16(0.18)$ & $14.12(0.16)$ & $<0.001$ \\
\hline & $\mathrm{n}(\%)$ & $\%(\mathrm{SE})$ & $\%(\mathrm{SE})$ & $\%(\mathrm{SE})$ & $\%(\mathrm{SE})$ & $\%(\mathrm{SE})$ & $p$-value \\
\hline $\begin{array}{c}\text { Male Sex } \\
\text { Race/Ethnicity }\end{array}$ & 3205 (48.49) & $49.39(0.67)$ & $40.14(2.28)$ & $36.76(3.24)$ & $56.72(1.48)$ & $47.01(1.44)$ & $<0.001$ \\
\hline Mexican American & $1041(15.75)$ & $9.49(1.12)$ & $8.02(1.09)$ & $8.97(2.29)$ & $9.06(1.10)$ & $10.78(1.30)$ & \multirow{6}{*}{$<0.001$} \\
\hline Other Hispanic & $731(11.06)$ & $6.49(0.79)$ & $6.87(1.34)$ & $7.29(1.75)$ & $6.99(0.85)$ & $5.66(0.65)$ & \\
\hline NH White & $2353(35.60)$ & $63.36(1.98)$ & $68.02(2.66)$ & $48.17(4.57)$ & $67.21(1.89)$ & $59.23(2.44)$ & \\
\hline NH Black & $1376(20.82)$ & 11.29 (1.11) & $6.77(0.98)$ & $30.32(3.53)$ & $3.54(0.52)$ & $18.81(1.89)$ & \\
\hline NH Asian & 849 (12.84) & $5.55(0.52)$ & $7.45(0.76)$ & $2.15(0.63)$ & $8.97(0.97)$ & $1.52(0.16)$ & \\
\hline Other/Multi-Racial & $260(3.93)$ & $3.83(0.40)$ & $2.88(0.58)$ & $3.10(1.01)$ & $4.24(0.59)$ & $4.00(0.61)$ & \\
\hline Low SES & 1355 (22.69) & $15.43(1.05)$ & $13.10(1.46)$ & $16.56(1.99)$ & $15.22(1.23)$ & $16.67(1.47)$ & 0.143 \\
\hline CKD & $966(14.61)$ & $12.07(0.52)$ & $6.31(1.05)$ & $3.60(1.05)$ & $11.73(0.77)$ & $16.70(0.83)$ & $<0.001$ \\
\hline Physically Active & $2317(69.98)$ & $69.38(1.08)$ & $77.93(1.83)$ & $76.24(3.30)$ & 67.79 (1.98) & $62.88(2.03)$ & $<0.001$ \\
\hline Smoker & $2981(45.10)$ & $46.28(1.26)$ & $37.38(2.46)$ & $40.78(3.55)$ & 49.76 (1.57) & 48.09 (1.43) & $<0.001$ \\
\hline Glucose Medication & $797(12.06)$ & $9.14(0.53)$ & 0 & 0 & $8.57(0.76)$ & $15.86(1.00)$ & $<0.001$ \\
\hline Cholesterol Medication & $1206(18.25)$ & $17.27(0.67)$ & 0 & 0 & $21.64(1.20)$ & $24.29(1.31)$ & 0.158 \\
\hline Hypertension Medication & $1678(25.39)$ & $22.09(0.88)$ & 0 & 0 & $23.23(1.47)$ & $35.69(1.51)$ & $<0.001$ \\
\hline
\end{tabular}

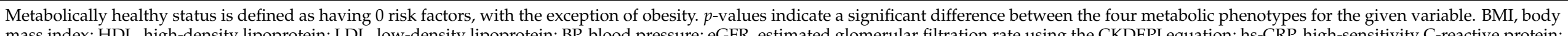

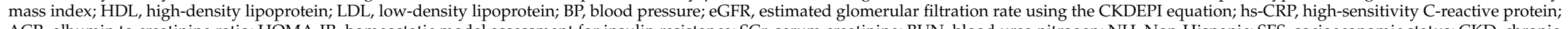

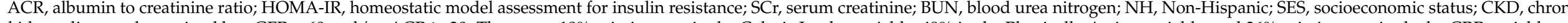

kidney disease, determined by eGFR $<60$ and/or ACR $\geq 30$. There was $18 \%$ missingness in the Caloric Intake variable, $49 \%$ in the Physically Active variable, and $36 \%$ missingness in the hs-CRP variable. 
The linear regression analyses in Table 3 utilized three consecutive models to demonstrate eGFR in the metabolic phenotypes ranging from a strict definition of metabolic health to the conventional definition outlined by the NCEP ATP III. The most conservative definition defined metabolic health as 0 risk factors with the exception of obesity, where the frequency of MHN was $19.11 \%$, MHO was $5.59 \%$, MUN was $38.40 \%$, and MUO was $36.90 \%$. When metabolic health was defined as one or fewer metabolic risk factors, the frequency of each phenotype shifted towards metabolically healthy: MHN accounted for $36.67 \%$ of the population, $\mathrm{MHO}$ was $15.56 \%$, MUN was $20.85 \%$, and MUO was $26.92 \%$. Further shifts towards the metabolically healthy phenotypes were demonstrated when metabolic health was defined as two or fewer metabolic risk factors: MHN accounted for $48.64 \%$ of the population, $\mathrm{MHO}$ was $27.36 \%$, MUN was $8.88 \%$, and MUO was $15.12 \%$. Across models, the eGFR in the MHO phenotype was slightly higher than that of the reference although this association was not found to be significantly different. The MUN and MUO phenotypes had significantly lower eGFR than the reference group (MHN). Across all three models, the MUN phenotype consistently demonstrated the lowest average eGFR compared to all other phenotypes. This finding is consistent with the demographic information represented in Table 2.

Table 3. Linear Regression Analyses.

\begin{tabular}{ccccccc}
\hline \multirow{2}{*}{ Coefficient } & \multicolumn{2}{c}{ Model 1 $^{\mathbf{a}}$} & \multicolumn{2}{c}{ Model 2 $^{\mathbf{b}}$} & \multicolumn{2}{c}{ Model 3 $^{\mathbf{c}}$} \\
\cline { 2 - 7 } & $\boldsymbol{B}$ & SE $\boldsymbol{B}$ & $\boldsymbol{B}$ & SE B & B & SE B \\
\hline Intercept (MHN) & 103.93 & 0.91 & 101.98 & 0.79 & 99.43 & 0.73 \\
MHO & 2.50 & 1.42 & 2.03 & 1.15 & 1.54 & 0.80 \\
MUN & $-9.60^{* *}$ & 0.80 & $-12.30^{* *}$ & 1.09 & $-12.33^{* *}$ & 1.20 \\
MUO & $-8.74^{* *}$ & 0.96 & $-9.55^{* *}$ & 1.01 & $-10.53^{* *}$ & 0.96 \\
\hline$R^{2}$ & \multicolumn{2}{c}{0.042} & \multicolumn{2}{c}{0.077} & \multicolumn{2}{c}{0.059}
\end{tabular}

** $p<0.001$. ${ }^{\text {a }}$ Metabolic health defined as 0 metabolic abnormalities (with the exception of obesity) and 1 risk factor considered unhealthy. ${ }^{b}$ Metabolic health defined as 1 metabolic abnormality (with the exception of obesity) and 2 risk factors considered unhealthy. ${ }^{\mathrm{c}}$ Metabolic health defined as 2 metabolic abnormalities (with the exception of obesity) and 3 risk factors considered unhealthy.

Correlates of eGFR and SCr are demonstrated in Table 4. The risk factors found to be most closely associated with low renal function were systolic blood pressure (eGFR, $r=-0.250, p<0.01$, SCr $r=0.105, p<0.001$ ) and waist circumference (eGFR, $r=-0.175$, $p<0.01, \mathrm{SCr}, r=0.096, p<0.001)$. HDL demonstrated a significant negative association with SCr $(r=-0.123, p<0.001)$, indicating that as HDL increases, SCr decreases. In the MHO phenotype, which included individuals with no risk factors except obesity, the fasting triglyceride levels had a small inverse relationship with eGFR $(r=-0.159, p<0.05)$, and HDL, BMI, and waist circumference demonstrated small negative relationships with SCr $(r=-0.172,-0.165$, and -0.123 , respectively, $p<0.05$ for all $)$. Renal function in the MUN phenotype demonstrated significant correlations with systolic blood pressure (eGFR, $r=-0.269, p<0.01$, SCR, $r=0.106, p<0.001$ ), BMI (eGFR, $r=-0.124, p<0.001$, SCR, $r=0.061, p<0.05$ ), and waist circumference (eGFR, $r=-0.282, p<0.001, \mathrm{SCr}, r=0.187$, $p<0.001$ ). In the MUN group, eGFR and SCr were negatively correlated to HDL (eGFR, $r=-0.088, p<0.001$, SCr, $r=-0.126, p<0.01)$. 
Table 4. Correlates of eGFR.

\begin{tabular}{|c|c|c|c|c|c|c|}
\hline & Overall eGFR & Overall SCr & MHO eGFR & MHO SCr & MUN eGFR & MUN SCr \\
\hline $\mathrm{FG}, r$ & $-0.119 * *$ & $0.026^{*}$ & 0.015 & -0.023 & $-0.069^{* *}$ & -0.020 \\
\hline $\mathrm{n}$ & 6610 & 6588 & 367 & 366 & 2537 & 2529 \\
\hline $\mathrm{TG}, r$ & $-0.083^{* *}$ & 0.040 * & $-0.159 *$ & 0.042 & $-0.044 *$ & 0.034 \\
\hline $\mathrm{n}$ & 6610 & 6588 & 367 & 366 & 2537 & 2529 \\
\hline HDL, $r$ & -0.002 & $-0.123^{* *}$ & -0.065 & $-0.172 *$ & $-0.088^{* *}$ & $-0.126^{* *}$ \\
\hline $\mathrm{n}$ & 6610 & 6588 & 367 & 366 & 2537 & 2529 \\
\hline $\mathrm{SBP}, r$ & $-0.25^{* *}$ & $0.105^{* *}$ & 0.008 & 0.078 & $-0.269^{* *}$ & $0.106^{* *}$ \\
\hline $\mathrm{n}$ & 6610 & 6588 & 367 & 366 & 2537 & 2529 \\
\hline $\mathrm{DPB}, r$ & -0.023 & 0.011 & -0.084 & -0.067 & 0.00 & 0.020 \\
\hline $\mathrm{n}$ & 6610 & 6588 & 367 & 366 & 2537 & 2529 \\
\hline BMI, $r$ & $-0.056^{* *}$ & 0.011 & 0.049 & $-0.165 *$ & $-0.124^{* *}$ & 0.061 * \\
\hline $\mathrm{n}$ & 6610 & 6588 & 367 & 366 & 2537 & 2529 \\
\hline WC, $r$ & $-0.175^{* *}$ & $0.096^{* *}$ & -0.033 & $-0.123 *$ & $-0.282^{* *}$ & $0.187^{* *}$ \\
\hline $\mathrm{n}$ & 6445 & 6424 & 358 & 357 & 2481 & 2473 \\
\hline
\end{tabular}

${ }^{*} p<0.05,{ }^{* *} p<0.001$; SCr, serum creatinine; $r$, Pearson's Correlation Coefficient; $\mathrm{n}$, number of observations; eGFR, estimated glomerular filtration rate; FG, fasting glucose; TG, triglycerides; HDL, high-density lipoprotein; SBP, systolic blood pressure; DBP, diastolic blood pressure; BMI, body mass index; WC, waist circumference.

Figure 1 demonstrates the average eGFR in individuals with one, two, or three risk factors. This figure represents the impact of each metabolic risk factor, including obesity, on eGFR. The reference point was an individual with 0 risk factors $\left(e G F R=103.93 \mathrm{~mL} / \mathrm{min} / 1.73 \mathrm{~m}^{2}\right)$. Regardless of the number of risk factors an individual had, those with hypertension consistently had the lowest eGFR, and the eGFR in those with hypertension decreased as the number of risk factors increased. Dyslipidemia in the form of high fasting triglycerides was the second most detrimental risk factor associated with eGFR. Individuals with low HDL as defined by the NCEP ATP III criteria consistently demonstrated the highest eGFR despite this being a metabolic risk factor.

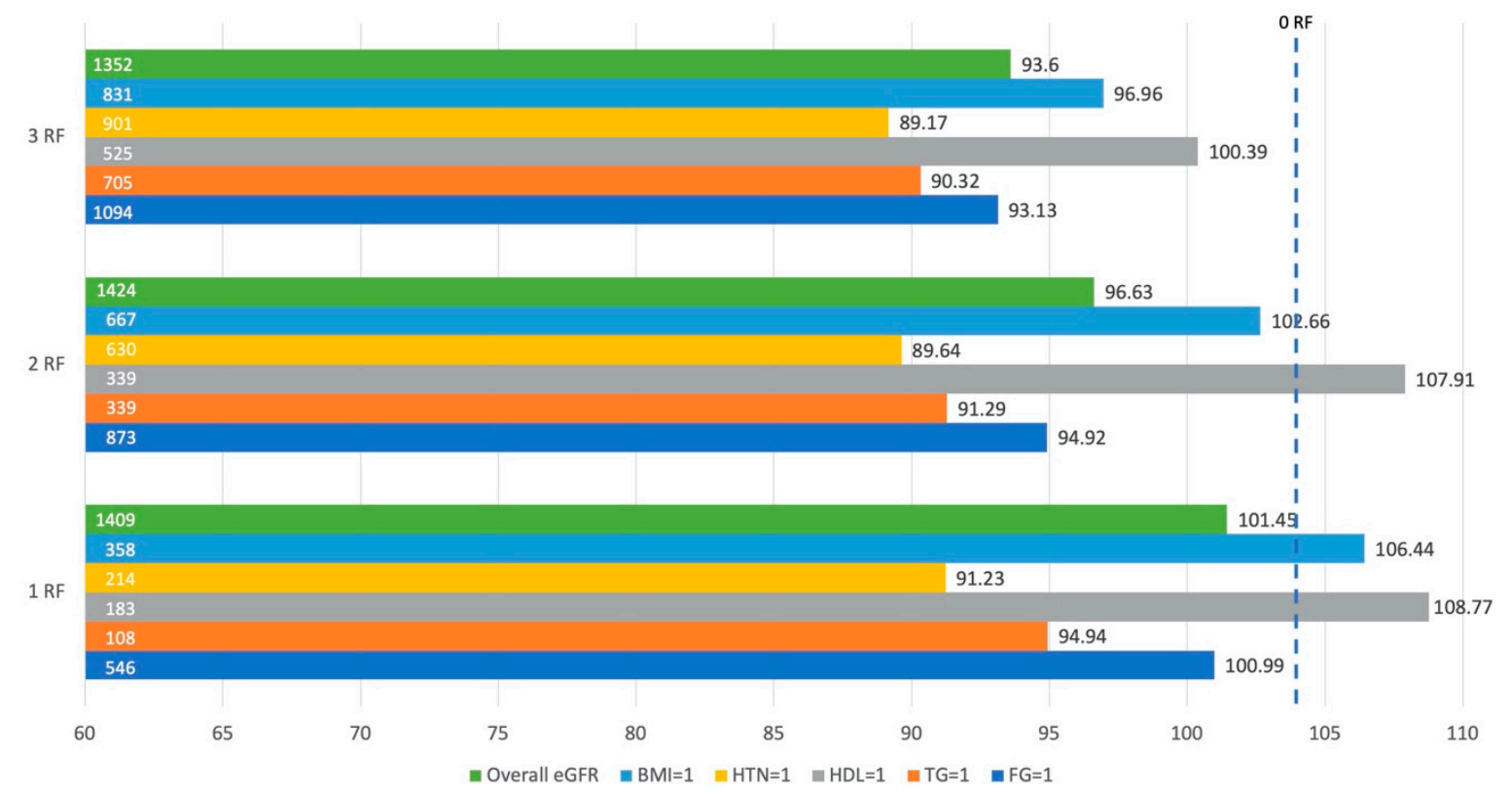

Figure 1. eGFR and Metabolic Risk Factors.

This figure represents the average estimated glomerular filtration rate (eGFR) in individuals with one, two, and three risk factors, including obesity. By highlighting each individual risk factor, we demonstrate what eGFR would be if an individual had a particular 
risk factor either independently or in conjunction with other risk factors. The numbers on the left side of the horizontal bars indicate the sample size, whereas the numbers to the right of each horizontal bar represent the eGFR for that condition. Overall eGFR is the average eGFR for individuals with one, two, or three risk factors; $\mathrm{BMI}=1$ indicates presence of obesity; HTN $=1$ indicates hypertension; $\mathrm{HDL}=1$ indicates dyslipidemia as determined by the high-density lipoprotein variable; TG $=1$ indicates dyslipidemia as determined by fasting triglycerides; and $\mathrm{FG}=1$ indicates high fasting glucose. The eGFR for the reference group ( 0 risk factors) is $103.93 \mathrm{~mL} / \mathrm{min} / 1.73 \mathrm{~m}^{2}$.

\section{Discussion}

The purpose of the present study was to report the prevalence of the strict metabolic phenotypes, renal function in each phenotype, and the risk factors associated with renal function. Our primary outcomes indicate that the strictly defined MUN phenotype accounted for the largest proportion of the U.S. population, whereas the MHO phenotype accounted for the smallest. In previous studies using the same strict definition of metabolic health, the MUN phenotype varied from $35-45 \%$ of the population, and the MHO phenotype ranged from $2.5-5.5 \%$ of the population, on average $[11,12,23,34]$. In the present study, the proportions of the "intriguing" phenotypes fall within the purviews of prior research. Similar overall results can be seen in previous studies $[12,34]$ although the MHN and MUN populations can vary widely depending on the population measured. Kouvari et al. reported a large percentage of the MHN phenotype (36.30\%) in the relatively homogenous Greek population assessed in the ATTICA cohort study [11], which is almost double the frequency of the MHN in the present study. Our prior research identified a large percentage of the MUO phenotype (57.79\%) in a federally qualified health center in the southern U.S. [23], which is 1.5 times the proportion that we established here. The sample used in the present study is representative of the entire U.S. population and therefore consists of greater racial and ethnic diversity than the study by Kouvai et al. as well as greater socioeconomic and geographic diversity than our prior study.

In the study sample, renal function was lowest in the MUN phenotype. However, it is important to note that CKD was more prevalent in the MUO phenotype due to the definition of CKD and the high ACR $\left(A=\pi r^{2}=44.45 \mathrm{mg} / \mathrm{g}, \mathrm{SE}=6.20\right)$ in the MUO phenotype. These findings persisted across multiple definitions of metabolic health ranging from the strict definition to the standard definition of MetS, demonstrating that one metabolic risk factor may be similarly indicative of renal dysfunction as two or three risk factors but that CKD status was more highly dependent upon ACR than eGFR. The MUN phenotype, while not typically perceived as high risk [10], has been correlated with adverse health outcomes, such as poor renal function [23], type 2 diabetes, cardiovascular events, and mortality [35]. In our study as well as previously reported findings [17,23], the MUN phenotype was correlated with older age. CKD has also been reported to be more common in individuals of older age [7], though this finding may be due to the prolonged presence of metabolic risk factors rather than age itself. A recent pilot study by Valdez et al. demonstrated that renal health was independent of age in individuals with no metabolic risk factors [36]. Still, more research is warranted to assess the renal risk in individuals with one or more metabolic risk factors and normal weight given that this constitutes a majority of the U.S. population.

Overweight $(25 \leq \mathrm{BMI} \leq 30)$ and obese $(\mathrm{BMI} \geq 30)$ individuals have a $40 \%$ to $80 \%$ increased risk of CKD, respectively [37]. However, in the present study, the MHO phenotype presented with renal function that was comparable to the reference group (MHN). Similar to previous findings [38], the MHO phenotype was younger in age, indicating that the findings could be attributed to the short amount of time that these individuals have been in an obese state. In the early stages of obesity, the kidneys engage in compensatory vasodilation and hyperfiltration in an attempt to maintain sodium balance despite increased tubular sodium reabsorption [39]. Over time, the high-pressure system caused by hyperfiltration causes glomerulosclerosis, which may not be detectable via changes in serum creatinine values until renal function has decreased by approximately $50 \%$ [40]. The higher eGFR 
demonstrated in the MHO phenotype presents a phenomenon that may be explained by the transient state of "healthy obesity" wherein the detrimental metabolic effects of the obese state have not yet had time to manifest [11]. This finding demonstrates the inadequacy of BMI as a proxy measure for body composition, warranting future research on the relationship between body composition and renal function.

Long-term studies have demonstrated higher risk of CVD and mortality in the MHO phenotype [41,42]. Additionally, a longitudinal study by Kouvari et al. demonstrated that $52 \%$ of individuals classified as MHO transitioned to the MUO status within a 10-year timeframe [11]. While we cannot determine chronicity of disease in the present crosssectional sample, we did observe possible indicators of future disease. A high hs-CRP level was detected in the obese phenotypes, which is indicative of systemic inflammation likely due to excess adipose tissue $[43,44]$. Additionally, the lipid profile of the MHO phenotype was within normal range yet inferior to that of the reference group. On average, triglycerides and LDL were 10 points higher than the MHN phenotype, and HDL was 5 points lower, increasing the risk of future CKD $[45,46]$. Although individuals classified as $\mathrm{MHO}$ have healthy metabolic and renal markers in cross-sectional analyses [47], it is likely that the inflammatory process of persistent obesity will be followed by metabolic risk factors and eventual declines in renal health. Further research is warranted to investigate the specific conditions necessary to maintain metabolic health in the presence of obesity.

In the overall sample, we found HTN, a high WC, and high fasting glucose to be negatively correlated with eGFR, which is intuitive given that hypertension and hyperglycemia are the two main precursors of CKD in the developed world [7]. In the MUN phenotype, eGFR had the largest correlations with HTN and WC. While these individuals were not obese as classified by BMI cutoffs, they did demonstrate a WC that was approximately $10 \mathrm{~cm}$ greater than that of the MHN, indicating that they carry more of their weight in the central region of their body. Central adiposity in the form of visceral adipose tissue (VAT) has been identified as a major contributor of insulin resistance [26] and is more metabolically active than subcutaneous fat or adipose tissue carried in the lower limbs [48]. The metabolically active VAT is possibly a major contributor to the metabolically unhealthy status and reduced renal function observed in this phenotype.

Unique to our study, HDL had a small negative association with eGFR, indicating that lower levels of HDL were correlated with a higher eGFR. This was demonstrated in Figure 1, where individuals with low HDL as one of their risk factors had a higher average eGFR than individuals with any other risk factor. The Pearson's correlation analyses were consistent with these findings except for the MUN group, which displayed conflicting findings-indicating that eGFR increased as HDL decreased, but SCr increased while HDL decreased. The findings that higher HDL levels may increase risk of CKD contradict many previous research findings $[38,45,46,49]$, but there have also been studies that confirmed greater risk of mortality associated with high HDL levels [50]. It is possible that the weak negative correlation demonstrated in our study could be explained by outliers with rare genetic variations in HDL receptors [51] or high levels of inflammation [52]. In future investigations, HDL function may prove to be more important than quantity. Still, further research should be done to understand these findings.

\section{Strengths and Limitations}

This study is the first to utilize a strict definition of metabolic health in the assessment of CKD while also utilizing NHANES complex survey sample weighting techniques. Much of the research in metabolic phenotypes and renal function is conducted in Asian populations, whereas our sample was taken from a racially and ethnically diverse population in the U.S. The large sample size and use of the complex survey sample weighting techniques allowed us to report unique findings that are representative of the U.S. population. This study was limited by the cross-sectional nature of the data, which prevents us from making inferences about the temporal sequence of events leading to declines in renal function. NHANES sampling techniques and measures are widely accepted, yet selection 
bias may still occur. For example, 18 individuals who met the inclusion criteria of the present study reported dialysis in the past year. Given the voluntary nature of research, it is likely that few of the ill and/or infirmed individuals selected for this study chose to participate. To marginally correct for this, the sample weighting procedures adjust for nonresponse to reduce potential bias. The sample sizes of the four phenotypes varied widely, and the MHO phenotype was very small (5.59\% of the population), lowering the statistical power in comparisons made using this phenotype. Additionally, the amount of variance explained by each of the regression models, demonstrated by the R2 values, was very low. A larger amount of variance could be explained by including variables such as age, sex, and race/ethnicity, but these values were considered in the equation estimating GFR and therefore were not added to the regression models. Metabolic risk factors, drug information, and BMI were considered in the metabolic phenotypes and therefore were not added to the regression equation. SES and caloric intake were not statistically different among the four phenotypes, and there was a large percentage of missingness in the PA, smoking, and hs-CRP variables, excluding these variables from the regression analyses. Therefore, the regression models are presented with the unadjusted results, which explains a small percentage of the variance in renal function yet demonstrates significant differences between the phenotypes. Glomerular filtration rate was estimated using an equation that utilizes serum creatinine, which can be affected by muscle mass, muscle breakdown, exercise, nutrition, medications, and hydration status. We were limited to one-time measures of eGFR and hs-CRP due to the cross-sectional nature of the study. To diagnose CKD, measures of $\mathrm{SCr}$ should be taken twice, approximately three months apart. Measures of hs-CRP should also be taken twice, approximately two weeks apart, to obtain an average measure of inflammation.

\section{Conclusions}

In the present study, we utilized a complex survey sample weighting technique to identify a sizable frequency of individuals with metabolic risk factors and/or obesity in the U.S. population. We observed higher proportions of males and individuals of older age in the metabolically unhealthy phenotypes, whereas in the obese phenotypes, we observed higher proportions of non-Hispanic Black individuals and greater levels of inflammation represented by hs-CRP values above $3.0 \mathrm{mg} / \mathrm{L}$. Using a strict definition of metabolic health, we found that renal function was lowest in the MUN phenotype. These findings persisted when using more lenient definitions of metabolic health. The renal health of the MHO phenotype was not statistically different from the reference group; however, these findings are likely transient given previous reports from longitudinal studies. Hypertension, waist circumference, and HDL were negatively correlated with renal function, implicating future research in the area of dyslipidemia and renal function.

Author Contributions: Conceptualization, K.E.A., R.G.B., L.K.F. and K.R.Y.; methodology, K.E.A., J.S.F., R.G.B. and K.R.Y.; software, K.E.A. and K.R.Y.; formal analysis, K.E.A., R.G.B. and K.R.Y.; investigation, K.E.A., R.G.B. and K.R.Y.; resources, L.K.F. and J.S.F.; data curation, K.E.A., R.G.B. and K.R.Y.; writing - original draft preparation, K.E.A., R.G.B. and K.R.Y.; writing-review and editing, K.E.A., L.K.F., J.S.F., R.G.B. and K.R.Y.; visualization, K.E.A., R.G.B. and K.R.Y.; supervision, R.G.B. and K.R.Y.; project administration, K.E.A.; funding acquisition, R.G.B. All authors have read and agreed to the published version of the manuscript.

Funding: The APC was funded by Brown Foundation Fund, Baylor University.

Institutional Review Board Statement: Ethical review and approval were waived for this study due to the secondary nature of the CDC datasets.

Informed Consent Statement: Patient consent was waived due to the nature of the secondary datasets. The consent documents are posted on the CDC website.

Data Availability Statement: All data are available from the CDC at https:/ /wwwn.cdc.gov/nchs/ nhanes/. 
Acknowledgments: Luke Richardson, Ron Wilson, Burritt Hess, Matthew Rafalski, Sally Weaver, and all faculty and staff at Baylor University who have made this work possible.

Conflicts of Interest: The authors declare no conflict of interest. The funders had no role in the design of the study; in the collection, analyses, or interpretation of data; in the writing of the manuscript, or in the decision to publish the results.

\section{References}

1. United States Renal Data System. 2018 USRDS Annual Data Report: Epidemiology of Kidney Disease in the United States; National Institutes of Health, National Institute of Diabetes and Digestive and Kidney Diseases: Bethesda, MD, USA, 2018.

2. Jha, V.; Garcia-Garcia, G.; Iseki, K.; Li, Z. Chronic Kidney Disease: Global Dimension and Perspectives. Lancet 2013, 382, $260-272$. [CrossRef]

3. Saran, R.; Robinson, B.; Abbott, K. US Renal Data System 2017 Annual Data Report: Epidemiology of Kidney Disease in the United States. Am. J. Kidney Dis. 2018, 71 (Suppl. S1), A7. [CrossRef] [PubMed]

4. Keith, D.S.; Nichols, G.A.; Gullion, C.M.; Brown, J.B.; Smith, D.H. Longitudinal Follow-up and Outcomes Among a Population With Chronic Kidney Disease in a Large Managed Care Organization. Arch. Intern. Med. 2004, 164, 659-663. [CrossRef] [PubMed]

5. Moore, J.X. Metabolic Syndrome Prevalence by Race/Ethnicity and Sex in the United States, National Health and Nutrition Examination Survey, 1988-2012. Prev. Chronic Dis. 2017, 14, E24. [CrossRef] [PubMed]

6. Hales, C.; Carroll, M.; Fryar, C.; Ogden, C. Prevalence of Obesity among Adults and Youth: United States, 2017-2018; National Center for Health Statistics: Hyattsville, MD, USA, 2020.

7. Centers for Disease Control and Prevention. Chronic Kidney Disease in the United States, 2019; US Department of Health and Human Services, Centers for Disease Control and Prevention: Atlanta, GA, USA, 2019.

8. Weisinger, J.R.; Kempson, R.L.; Eldridge, F.L.; Swenson, R.S. The Nephrotic Syndrome: A Complication of Massive Obesity. Ann. Intern. Med. 1974, 81, 440-447. [CrossRef] [PubMed]

9. Yarnoff, B.O.; Hoerger, T.J.; Shrestha, S.S.; Simpson, S.K.; Burrows, N.R.; Anderson, A.H.; Xie, D.; Chen, H.-Y.; Pavkov, M.E.; the CRIC Study Investigators. Modeling the Impact of Obesity on the Lifetime Risk of Chronic Kidney Disease in the United States Using Updated Estimates of GFR Progression from the CRIC Study. PLoS ONE 2018, 13, e0205530. [CrossRef] [PubMed]

10. Alizadeh, S.; Esmaeili, H.; Alizadeh, M.; Daneshzad, E.; Sharifi, L.; Radfar, H.; Radaei, M.K. Metabolic Phenotypes of Obese, Overweight, and Normal Weight Individuals and Risk of Chronic Kidney Disease: A Systematic Review and Meta-Analysis. Arch. Endocrinol. Metab. 2019, 63, 427-437. [CrossRef]

11. Kouvari, M.; Panagiotakos, D.B.; Yannakoulia, M.; Georgousopoulou, E.; Critselis, E.; Chrysohoou, C.; Tousoulis, D.; Pitsavos, C.; ATTICA Study Investigators. Transition From Metabolically Benign to Metabolically Unhealthy Obesity and 10-Year Cardiovascular Disease Incidence: The ATTICA Cohort Study. Metabolism 2019, 93, 18-24. [CrossRef]

12. Adair, K.E.; Padgett, R.N.; von Waaden, N.; Wilson, R.L.; Bowden, R.G. Metabolic Health, Obesity, and Cardiovascular Disease: 2015-2016 National Health and Nutrition Examination Survey. Am. J. Med. Sci. 2020, 361, 244-252. [CrossRef]

13. Van Vliet-Ostaptchouk, J.V.; Nuotio, M.-L.; Slagter, S.N.; Doiron, D.; Fischer, K.; Foco, L.; Gaye, A.; Gögele, M.; Heier, M.; Hiekkalinna, T.; et al. The Prevalence of Metabolic Syndrome and Metabolically Healthy Obesity in Europe: A Collaborative Analysis of Ten Large Cohort Studies. BMC Endocr. Disord. 2014, 14, 9. [CrossRef]

14. Caleyachetty, R.; Thomas, G.N.; Toulis, K.A.; Mohammed, N.; Gokhale, K.M.; Balachandran, K.; Nirantharakumar, K. Metabolically Healthy Obese and Incident Cardiovascular Disease Events Among 3.5 Million Men and Women. J. Am. Coll. Cardiol. 2017, 70, 1429-1437. [CrossRef]

15. Wildman, R.P.; Muntner, P.; Reynolds, K.; McGinn, A.P.; Rajpathak, S.; Wylie-Rosett, J.; Sowers, M.R. The Obese Without Cardiometabolic Risk Factor Clustering and the Normal Weight With Cardiometabolic Risk Factor Clustering: Prevalence and Correlates of 2 Phenotypes Among the US Population (NHANES 1999-2004). Arch. Intern. Med. 2008, 168, 1617-1624. [CrossRef] [PubMed]

16. Nam, K.H.; Yun, H.-R.; Joo, Y.S.; Kim, J.; Lee, S.; Lee, C.; Park, K.S.; Park, J.T.; Chang, T.-I.; Kang, E.W.; et al. Changes in Obese Metabolic Phenotypes over Time and Risk of Incident Chronic Kidney Disease. Diabetes Obes. Metab. 2018, 20, $2778-2791$. [CrossRef]

17. Bradshaw, P.T.; Monda, K.L.; Stevens, J. Metabolic Syndrome in Healthy Obese, Overweight, and Normal Weight Individuals: The Atherosclerosis Risk in Communities Study. Obesity 2013, 21, 203-209. [CrossRef]

18. Yun, H.-R.; Kim, H.; Park, J.T.; Chang, T.I.; Yoo, T.-H.; Kang, S.-W.; Choi, K.H.; Sung, S.; Kim, S.W.; Lee, J.; et al. Obesity, Metabolic Abnormality, and Progression of CKD. Am. J. Kidney Dis. 2018, 72, 400-410. [CrossRef]

19. St-Onge, M.-P.; Janssen, I.; Heymsfield, S.B. Metabolic Syndrome in Normal-Weight Americans: New Definition of the Metabolically Obese, Normal-Weight Individual. Diabetes Care 2004, 27, 2222-2228. [CrossRef] [PubMed]

20. Zhang, J.; Jiang, H.; Chen, J. Combined Effect of Body Mass Index and Metabolic Status on the Risk of Prevalent and Incident Chronic Kidney Disease: A Systematic Review and Meta-Analysis. Oncotarget 2016, 8, 35619-35629. [CrossRef]

21. Lavie, C.J.; Laddu, D.; Arena, R.; Ortega, F.B.; Alpert, M.A.; Kushner, R.F. Healthy Weight and Obesity Prevention: JACC Health Promotion Series. J. Am. Coll. Cardiol. 2018, 72, 1506-1531. [CrossRef] [PubMed] 
22. Diniz, M.d.F.H.S.; Beleigoli, A.M.R.; Ribeiro, A.L.P.; Vidigal, P.G.; Bensenor, I.M.; Lotufo, P.A.; Duncan, B.B.; Schmidt, M.I.; Barreto, S.M. Factors Associated with Metabolically Healthy Status in Obesity, Overweight, and Normal Weight at Baseline of ELSA-Brasil. Medicine 2016, 95, e4010. [CrossRef] [PubMed]

23. Adair, K.E.; von Waaden, N.; Rafalski, M.; Hess, B.W.; Weaver, S.P.; Bowden, R.G. Metabolic Phenotypes and Chronic Kidney Disease: A Cross-Sectional Assessment of Patients from a Large Federally Qualified Health Center. Life 2021, 11, 175. [CrossRef] [PubMed]

24. Chen, T.; Clark, J.; Riddles, M.; Mohadjer, L.; Fakhouri, T. National Health and Nutrition Examination Survey, 2015-2018: Sample Design and Estimation Procedures; National Center for Health Statistics: Paris, France, 2020.

25. Chen, T.; Parker, J.; Clark, J.; Shin, H.; Rammon, J.; Burt, V. National Health and Nutrition Examination Survey: Estimation Procedures, 2011-2014; National Center for Health Statistics: Hyattsville, MD, USA, 2018.

26. Grundy, S.M.; Cleeman, J.I.; Daniels, S.R.; Donato, K.A.; Eckel, R.H.; Franklin, B.A.; Gordon, D.J.; Krauss, R.M.; Savage, P.J.; Smith, S.C.; et al. Diagnosis and Management of the Metabolic Syndrome. Circulation 2005, 112, 2735-2752. [CrossRef]

27. Levey, A.S.; Stevens, L.A.; Schmid, C.H.; Zhang, Y.; Castro, A.F.; Feldman, H.I.; Kusek, J.W.; Eggers, P.; Van Lente, F.; Greene, T.; et al. A New Equation to Estimate Glomerular Filtration Rate. Ann. Intern. Med. 2009, 150, 604. [CrossRef]

28. Kidney Disease: Improving Global Outcomes (KDIGO) CKD Work Group. KDIGO 2012 Clinical Practice Guideline for the Evaluation and Management of Chronic Kidney Disease. Kidney Int. Suppl. 2013, 3, 1-150.

29. Centers for Disease Control and Prevention (CDC); National Center for Health Statistics (NCHS). National Health and Nutrition Examination Survey Data; U.S. Department of Health and Human Services, Centers for Disease Control and Prevention: Hyattsville, MD, USA, 2019. Available online: https:/ /Wwwn.Cdc.Gov/Nchs/Nhanes/ (accessed on 12 February 2021).

30. Physical Activity Guidelines for Americans. Physical Activity Guidelines Advisory Committee Report, 2019; US Department of Health and Human Services: Washington, DC, USA, 2018.

31. Fang, J.; Zhang, Z.; Ayala, C.; Thompson-Paul, A.M.; Loustalot, F. Cardiovascular Health Among Non-Hispanic Asian Americans: NHANES, 2011-2016. J. Am. Heart Assoc. 2019, 8, e011324. [CrossRef]

32. Friedewald, W.T.; Levy, R.I.; Fredrickson, D.S. Estimation of the Concentration of Low-Density Lipoprotein Cholesterol in Plasma, Without Use of the Preparative Ultracentrifuge. Clin. Chem. 1972, 18, 499-502. [CrossRef]

33. Matthews, D.R.; Hosker, J.P.; Rudenski, A.S.; Naylor, B.A. Homeostasis Model Assessment: Insulin Resistance and Beta-Cell Function from Fasting Plasma Glucose and Insulin Concentrations in Man. Diabetologia 1985, 28, 412. [CrossRef]

34. Chen, W.; Abramowitz, M.K. Metabolic Acidosis and the Progression of Chronic Kidney Disease. BMC Nephrol. 2014, 15, 55. [CrossRef]

35. Aung, K.; Lorenzo, C.; Hinojosa, M.A.; Haffner, S.M. Risk of Developing Diabetes and Cardiovascular Disease in Metabolically Unhealthy Normal-Weight and Metabolically Healthy Obese Individuals. J. Clin. Endocrinol. Metab. 2014, 99, 462-468. [CrossRef] [PubMed]

36. Valadez, E.; Buckley, D.; Ismaeel, A.; OLIVER, A.; Adair, K.; Papoutsi, E.; Prezioso, K.; Stamatis, A.; Koutakis, P.; Forsse, J. Is Age an Independent Factor in Assessing Renal Health and Function in Healthy Individuals? A Pilot Study. Int. J. Exerc. Sci. Conf. Proc. 2020, 2, 75 .

37. Wang, Y.; Chen, X.; Song, Y.; Caballero, B.; Cheskin, L.J. Association between Obesity and Kidney Disease: A Systematic Review and Meta-Analysis. Kidney Int. 2008, 73, 19-33. [CrossRef] [PubMed]

38. Jung, C.H.; Lee, M.J.; Kang, Y.M.; Hwang, J.Y.; Kim, E.H.; Park, J.-Y.; Kim, H.-K.; Lee, W.J. The Risk of Chronic Kidney Disease in a Metabolically Healthy Obese Population. Kidney Int. 2015, 88, 843-850. [CrossRef] [PubMed]

39. Hall, J.E.; Henegar, J.R.; Dwyer, T.M.; Liu, J.; Da Silva, A.A.; Kuo, J.J.; Tallam, L. Is Obesity a Major Cause of Chronic Kidney Disease? Adv. Ren. Replace. Ther. 2004, 11, 41-54. [CrossRef] [PubMed]

40. Gounden, V.; Bhatt, H.; Jialal, I. Renal Function Tests. In StatPearls; StatPearls Publishing: Treasure Island, FL, USA, 2021.

41. Ärnlöv, J.; Ingelsson, E.; Sundström, J.; Lind, L. Impact of Body Mass Index and the Metabolic Syndrome on the Risk of Cardiovascular Disease and Death in Middle-Aged Men. Circulation 2010, 121, 230. [CrossRef] [PubMed]

42. Kuk, J.L.; Ardern, C.I. Are Metabolically Normal but Obese Individuals at Lower Risk for All-Cause Mortality? Diabetes Care 2009, 32, 2297-2299. [CrossRef]

43. Saltiel, A.R.; Olefsky, J.M. Inflammatory Mechanisms Linking Obesity and Metabolic Disease. J. Clin. Investig. 2017, 127, 1-4. [CrossRef] [PubMed]

44. Cinti, S.; Mitchell, G.; Barbatelli, G.; Murano, I.; Ceresi, E.; Faloia, E.; Wang, S.; Fortier, M.; Greenberg, A.S.; Obin, M.S. Adipocyte Death Defines Macrophage Localization and Function in Adipose Tissue of Obese Mice and Humans. J. Lipid Res. 2005, 46, 2347-2355. [CrossRef]

45. Modification of Diet in Renal Disease Study Group; Hunsicker, L.G.; Adler, S.; Caggiula, A.; England, B.K.; Greene, T.; Kusek, J.W.; Rogers, N.L.; Teschan, P.E.; Beck, G. Predictors of the Progression of Renal Disease in the Modification of Diet in Renal Disease Study. Kidney Int. 1997, 51, 1908-1919. [CrossRef]

46. Muntner, P.; Coresh, J.; Smith, J.C.; Eckfeldt, J.; Klag, M.J. Plasma Lipids and Risk of Developing Renal Dysfunction: The Atherosclerosis Risk in Communities Study. Kidney Int. 2000, 58, 293-301. [CrossRef]

47. Chen, S.; Zhou, S.; Wu, B.; Zhao, Y.; Liu, X.; Liang, Y.; Shao, X.; Holthöfer, H.; Zou, H. Association between Metabolically Unhealthy Overweight/Obesity and Chronic Kidney Disease: The Role of Inflammation. Diabetes Metab. 2014, 40, 423-430. [CrossRef] 
48. Goodpaster, B.H.; Krishnaswami, S.; Harris, T.B.; Katsiaras, A.; Kritchevsky, S.B.; Simonsick, E.M.; Nevitt, M.; Holvoet, P.; Newman, A.B. Obesity, Regional Body Fat Distribution, and the Metabolic Syndrome in Older Men and Women. Arch. Intern. Med. 2005, 165, 777. [CrossRef]

49. Thomas, G.; Sehgal, A.R.; Kashyap, S.R.; Srinivas, T.R.; Kirwan, J.P.; Navaneethan, S.D. Metabolic Syndrome and Kidney Disease: A Systematic Review and Meta-Analysis. Clin. J. Am. Soc. Nephrol. 2011, 6, 2364-2373. [CrossRef]

50. Madsen, C.M.; Varbo, A.; Nordestgaard, B.G. Extreme High High-Density Lipoprotein Cholesterol Is Paradoxically Associated with High Mortality in Men and Women: Two Prospective Cohort Studies. Eur. Heart J. 2017, 38, 2478-2486. [CrossRef] [PubMed]

51. Zanoni, P.; Khetarpal, S.A.; Larach, D.B.; Hancock-Cerutti, W.F.; Millar, J.S.; Cuchel, M.; DerOhannessian, S.; Kontush, A.; Surendran, P.; Saleheen, D.; et al. Rare Variant in Scavenger Receptor BI Raises HDL Cholesterol and Increases Risk of Coronary Heart Disease. Science 2016, 351, 1166-1171. [CrossRef]

52. Corsetti, J.P.; Ryan, D.; Rainwater, D.L.; Moss, A.J.; Zareba, W.; Sparks, C.E. Cholesteryl Ester Transfer Protein Polymorphism (TaqIB) Associates With Risk in Postinfarction Patients With High C-Reactive Protein and High-Density Lipoprotein Cholesterol Levels. Arterioscler. Thromb. Vasc. Biol. 2010, 30, 1657-1664. [CrossRef] [PubMed] 